Теорія Ймовір. та Матем. Статист. Вип. 75, 2006
Theor. Probability and Math. Statist.

No. 75, 2007, Pages 41-50 S 0094-9000(08)00712-6

Article electronically published on January 23, 2008

\title{
THE STABILITY OF TRANSIENT QUASI-HOMOGENEOUS MARKOV SEMIGROUPS AND AN ESTIMATE OF THE RUIN PROBABILITY
}

UDC 519.21

\author{
M. V. KARTASHOV
}

\begin{abstract}
A time nonhomogeneous semigroup of Markov operators in a Banach space is called quasi-homogeneous if its infinitesimal operator has a dense domain and can be represented as the sum of the infinitesimal operator of a homogeneous semigroup and a bounded operator function.

We obtain estimates of the strong stability of a nonhomogeneous semigroup for the case where the underlying homogeneous semigroup is uniformly transient.
\end{abstract}

\section{INTRODUCTION}

The stability of perturbed homogeneous semigroups of operators is studied in the monograph 1 for discrete time. The foundations of the theory of time nonhomogeneous Markov processes are created in the books by E. B. Dynkin 2 and I. I. Gikhman and A. V. Skorokhod [3. The general theory of perturbations of operators can be found in [4.

In this paper, we develop an approach that originated in [5]. This approach is used in papers [5, 6] to estimate the stability of nonhomogeneous semigroups for which the underlying homogeneous semigroup is uniformly ergodic. In what follows we consider the transient case and solve the problem of stability.

As an example we apply the general results to estimate the ruin probability for a risk process with a time nonhomogeneous Poisson flow of insurance claims. Another approach to this problem for nonhomogeneous risk processes is described in [7].

\section{MAIn DEFinitions}

1. Let $(E, \Xi)$ be a measurable space. Denote by $f \Xi$ and $m \Xi$ the classes of all measurable functions and all finite charges on $(E, \Xi)$, respectively.

Assume that $\aleph \subset m \Xi$ is a Banach subspace of the space $m \Xi$ equipped with the norm $\|\cdot\|$.

Consider the space $\Im \subset f \Xi$ of functions, the Banach space that is dual to $\aleph$ and equipped with the norm

$$
\|f\|=\sup (|\mu f|,\|\mu\| \leq 1, \mu \in \aleph) .
$$

2000 Mathematics Subject Classification. Primary 60J45; Secondary 60A05.

Key words and phrases. Semigroup of operators, infinitesimal operator, uniform transiency, strong stability. 
The dual linear form is defined by

$$
\mu f=\int_{E} f(x) \mu(d x), \quad \mu \in \aleph, f \in \Im,
$$

in this case. Note also that

$$
\|\mu\|=\sup (|\mu f|,\|f\| \leq 1, f \in \Im) .
$$

Examples of the spaces and their dual spaces are given in [1, Chapter 1].

Every transition kernel $Q=(Q(x, B), x \in E, B \in \Xi)$ on $(E, \Xi)$ generates [5] the linear mappings

$$
\begin{gathered}
\mu Q(B)=\int_{E} \mu(d x) Q(x, B): m \Xi \rightarrow m \Xi, \\
Q f(x)=\int_{E} Q(x, d y) f(y): f \Xi \rightarrow f \Xi .
\end{gathered}
$$

The linear subclasses of these mappings equipped with finite norms

$$
\|Q\|=\sup (\|\mu Q\|,\|\mu\| \leq 1)=\sup (\|Q f\|,\|f\| \leq 1)<\infty
$$

are Banach spaces of linear bounded operators denoted by $L(\aleph)$ and $L(\Im)$, respectively. The product of the corresponding operators is generated by the kernel

$$
P Q(x, B)=\int_{E} P(x, d y) Q(y, B) .
$$

2. Let $(P(s, x, t, B), x \in E, B \in \Xi, 0 \leq s \leq t)$ be a Markov transition function [4, Chapter 3] understood in the wide sense. According to (2) and (3), one can associate the following mappings with this function:

$$
P_{s t}: m \Xi \rightarrow m \Xi, \quad P_{s t}: f \Xi \rightarrow f \Xi .
$$

If the mappings are bounded, then they generate a semigroup with respect to the multiplication [2, 3, 6. The mappings are bounded if $\aleph$ and $\Im$ are the spaces of all bounded charges equipped with the full variation norm and all bounded measurable functions equipped with the uniform norm, respectively.

3. A family of operators $\left(P_{s t}, 0 \leq s \leq t<\infty\right) \subset L(\Im)$ is called a bounded semigroup in $L(\Im)$ if

$$
P_{s u} P_{u t}=P_{s t}, \quad 0 \leq s \leq u \leq t, \quad \sup _{0 \leq s \leq t}\left\|P_{s t}\right\|<\infty .
$$

A semigroup of operators $\left(Q_{s t}, 0 \leq s \leq t<\infty\right)$ is time homogeneous if

$$
Q_{s t}=Q_{t-s}, \quad 0 \leq s \leq t, \quad Q_{s} Q_{t-s}=Q_{t} .
$$

If the limit

$$
\lim _{u \uparrow s, v \downarrow s}(v-u)^{-1}\left(P_{u v} f-f\right) \equiv A_{s} f, \quad s \geq 0,
$$

exists in the norm of the space $\Im$, then $A_{s}$ is called the infinitesimal operator of the nonhomogeneous semigroup $\left(P_{s t}\right)$.

Definition. A bounded semigroup $\left(P_{s t}, 0 \leq s \leq t<\infty\right)$ is called quasi-homogeneous if there exists a nontrivial bounded homogeneous semigroup $\left(Q_{t-s}, 0 \leq s \leq t<\infty\right)$ with the infinitesimal operator $A$ defined on a dense subspace $\Im_{0} \subset \Im$, namely if

$$
\text { for all } f \in \Im_{0}, \quad \text { there exists } \lim _{h \rightarrow 0} h^{-1}\left(Q_{h} f-f\right) \equiv A f
$$

and if, for some bounded family of operators $\left(D_{s}, 0 \leq s<\infty\right) \subset L(\Im)$, the infinitesimal operator $A_{s}$ of the semigroup $\left(P_{s t}\right)$ is given by
(AD)$$
A_{s}=A+D_{s} \quad \text { on } \Im_{0} .
$$ 
Remark. Condition (AD) is equivalent to

for all $s \geq 0$ and for all $f \in \Im_{0}$

(D)

$$
\text { the limit } \lim _{u \uparrow s, v \downarrow s}(v-u)^{-1}\left(P_{u v} f-Q_{v-u} f\right) \equiv D_{s} f \in \Im \quad \text { exists. }
$$

This follows directly from the definition of the infinitesimal operator and from the boundedness of $D_{s}$ (see [5]).

The semigroup $\left(Q_{t-s}\right)$ is called the underlying semigroup for $\left(P_{s t}\right)$.

The condition for the boundedness of the perturbation in the above definition can be written as follows:

$$
\sup _{s \leq t}\left\|D_{s}\right\|<\infty \text { for all } t>0 .
$$

4. A semigroup $\left(P_{s t}\right)$ is called uniformly transient (in the space $\Im$ ) if

$$
\left\|P_{s t}\right\| \rightarrow 0, \quad t \rightarrow \infty, \quad \text { for all } s \geq 0 .
$$

If a semigroup is homogeneous, that is, $P_{s t}=Q_{t-s}$, then the rate of convergence is geometrical:

$$
\text { there exists } \rho<1 \text { such that }\left\|Q_{t}\right\|=O\left(\rho^{t}\right), \quad t \rightarrow \infty .
$$

5. If a semigroup is quasi-homogeneous and uniformly transient, then

$$
\begin{gathered}
q_{T}(\beta) \equiv \sup _{t<T} \exp (\beta t)\left\|Q_{t}\right\| \\
\varepsilon_{T}(\beta) \equiv \sup _{t<T} \int_{0}^{t} \exp (\beta(t-u))\left\|D_{u} Q_{t-u}\right\| d u \\
\varepsilon_{T} \equiv \int_{0}^{T}\left\|D_{u}\right\| d u
\end{gathered}
$$

are finite for some $T \leq \infty$ and $\beta>0$.

The index $\varepsilon_{T}(\beta)$ can sometimes be not easy to evaluate. In this case, one can use the following upper bound:

$$
\varepsilon_{T}(\beta) \leq \varepsilon_{T} q_{T}(\beta) .
$$

Moreover, the measurability is necessary to construct the integral on the right hand side of (10). If this is not the case, then the index

$$
\varepsilon_{T}(\beta)=\sup _{t<T} \sup _{f \in \Im_{0}} \int_{0}^{t} \exp (\beta(t-u)) \frac{\left\|D_{u} Q_{t-u} f\right\|}{\|f\|} d u
$$

should be used instead of $\varepsilon_{T}(\beta)$ defined by (10). Indeed, the function $\left\|D_{u} Q_{t-u} f\right\|$ in (13) is Borel for all $f \in \Im_{0}$, since it is the limit of continuous functions,

$$
h^{-1}\left\|\left(P_{u-h, u}-Q_{h}\right) Q_{t-u} f\right\| \quad \text { as } h \downarrow 0
$$

(see [5]).

Note that inequality (12) is an obvious corollary of the inequality for the norm of the product of operators. The inequality between indices defined by (10) and (13) follows from the definition of the norm.

Throughout this paper, the integrals of operator-valued bounded functions are understood in the weak sense as integrals defined by their action for the elements $\mu \in \aleph$ and $f \in \Im$ :

$$
\mu\left(\int_{s}^{t} D_{u} d u\right) f=\int_{s}^{t} \mu D_{u} f d u
$$




\section{MAin RESUlts}

The following result contains a qualitative estimate of the stability of transient quasihomogeneous semigroups.

Theorem 1. Let $\left(P_{s t}, 0 \leq s \leq t<T\right)$ be a quasi-homogeneous bounded semigroup and let $T \leq \infty$. Assume that the underlying homogeneous semigroup $\left(Q_{t-s}\right)$ is uniformly transient in the sense that (9) holds. If the perturbation $\left(D_{s}\right)$ is such that

$$
\varepsilon_{T}(\beta)<1
$$

for some $\beta \geq 0$, then the semigroup $\left(P_{\text {st }}\right)$ also is uniformly transient and moreover

$$
\sup _{0 \leq s \leq t<T} \exp (\beta(t-s))\left\|P_{s, t}-Q_{t-s}\right\| \leq \varepsilon_{T}(\beta) \frac{q_{T}(\beta)}{1-\varepsilon_{T}(\beta)} .
$$

In particular, inequality (15) implies the bounds for the stability for functions $P_{s t} 1(x)$ viewed as survival probabilities on the interval from $s$ to $t$ for terminating semigroups. Here and in what follows, $1=1(x), x \in E$, stands for the identity mapping.

However there are sharper inequalities in this case. To state these results we note that $P_{s t} 1(x)$ are not increasing in $t$. Therefore the limits

$$
p_{s}(x)=\lim _{t \rightarrow \infty} P_{s t} 1(x), \quad \bar{p}(x)=\lim _{t \rightarrow \infty} Q_{t-s} 1(x)
$$

exist.

The functions $P_{s t} 1(x)$ are not increasing in $t$ in view of the semigroup and Markov properties:

$$
P_{s t} 1(x)-P_{s u} 1(x)=P_{s t}\left(1-P_{t u} 1\right)(x) \geq 0 \text { for all } s \leq t \leq u \text {. }
$$

Theorem 2. Let $\left(P_{s t}, 0 \leq s \leq t<\infty\right)$ be a quasi-homogeneous bounded semigroup. Then the limit functions (16) are such that

$$
\left\|p_{s}-\bar{p}\right\| \leq \int_{s}^{\infty}\left\|P_{s u}\right\|\left\|D_{u} \bar{p}\right\| d u .
$$

As an example we consider a risk process $\left(X_{t}, t \geq 0\right)$ that is a right continuous solution of the system

$$
\begin{gathered}
d X_{t}=c d t-d Z_{t}, \\
X_{0}=x>0,
\end{gathered}
$$

where $c>0$ is the premium intensity,

$$
Z_{t}=\sum_{k=1}^{\nu(t)} \xi_{k}
$$

$\xi_{n}$ are jointly independent identically distributed random variables representing the amounts of insurance payments, and $\nu(t)$ is an independent of $\left\{\xi_{n}\right\}$ and time nonhomogeneous Poisson process with intensity $\lambda_{t}$; that is,

$$
\begin{gathered}
P(\nu(t)-\nu(s)=n)=\left(\Lambda_{s}^{t}\right)^{n} \exp \left(-\Lambda_{s}^{t}\right) / n !, \quad s<t, \\
\Lambda_{s}^{t}=\int_{s}^{t} \lambda_{u} d u .
\end{gathered}
$$

We assume that $\lambda_{t}$ is a nonnegative measurable function on $\mathbb{R}_{+}$that is bounded on every finite interval.

We also assume that the process $\left(X_{t}\right)$ terminates at the moment $\tau_{x}$ when it enters the set $(-\infty, 0)$ (we say that $\tau_{x}$ is the ruin moment, $x=X_{0}$ ):

$$
\tau_{x}=\inf \left(t>0: X_{t} \notin[0, \infty)\right) .
$$


The infinitesimal operator of such a process is

$$
A_{t} f(x)=c f^{\prime}(x)+\lambda_{t} \int_{-\infty}^{x} f(x-y) d G(y)-\lambda_{t} f(x), \quad x \in \mathbb{R}_{+} .
$$

The operator $A_{t}$ is defined on $C_{1}\left(\mathbb{R}_{+}\right) \cap C_{b}\left(\mathbb{R}_{+}\right)$. Here and in what follows the common distribution function and moment generating function of the random variables $\xi_{n}$ are

$$
G(x)=\mathrm{P}\left(\xi_{1}<x\right), \quad \widehat{g}(t)=\mathrm{E} \exp \left(t \xi_{1}\right) .
$$

We further assume the Cramér condition:

$$
\text { there exists } \alpha>0 \text { such that } \widehat{g}(\alpha)<\infty \text {. }
$$

Along with $\left(X_{t}, t \geq 0\right)$ consider a similar time homogeneous process $\left(\bar{X}_{t}, t \geq 0\right)$ having the same premium intensity, insurance payments, and initial state $x$. The difference is that $\bar{X}_{t}$ has a constant claim intensity $\bar{\lambda}(t) \equiv \lambda$. This means that the corresponding Poisson process $\bar{\nu}(t)$ is homogeneous and has intensity $\lambda$. The parameter $\lambda>0$ used in the following inequalities is arbitrary, but the following nondegeneracy condition holds:

$$
\lambda \mathrm{E} \xi_{1}<c .
$$

One can define the same characteristics for the homogeneous process $\left(\bar{X}_{t}\right)$ as those for $\left(X_{t}\right)$. The characteristics of $\left(\bar{X}_{t}\right)$ are denoted with the same symbol as in the case of $\left(X_{t}\right)$ but with the bar.

The symbol $P_{s x}$ stands for the conditional probability given $X(s)=x$.

Theorem 3. Let $\left(X_{t}, 0 \leq t<T\right)$ be a nonhomogeneous risk process whose infinitesimal operator is given by (21) and let $\left(\bar{X}_{t}\right)$ be the corresponding homogeneous process with intensity $\lambda$ for which (24) holds.

If the constant $\alpha \geq 0$ is such that

$$
\beta \equiv \alpha c-\lambda(\widehat{g}(\alpha)-1) \geq 0,
$$

then

$$
\begin{aligned}
E_{\alpha} & \equiv \sup _{0 \leq s \leq t \leq T} \sup _{x \geq 0, B \in \Xi} e^{\alpha x+\beta(t-s)}\left|\mathrm{E}_{s x} e^{-\alpha X_{t}} 1_{\left\{X_{t} \in B\right\}}-\mathrm{E}_{s x} e^{-\alpha \bar{X}_{t}} 1_{\left\{\bar{X}_{t} \in B\right\}}\right| \\
& \leq \widehat{g}(\alpha) \int_{0}^{T}\left|\lambda_{u}-\lambda\right| \exp \left((\alpha c-\beta) \int_{0}^{u}\left(\lambda_{v}-\lambda\right) / \lambda d v\right) d u .
\end{aligned}
$$

Remark. Inequality (25) holds if and only if $0 \leq \alpha \leq \alpha_{L}$, where the Lundberg constant $\alpha_{L}>0$ is uniquely defined under conditions (24) and (23) by

$$
\alpha_{L}=\sup (\alpha>0: \alpha c-\lambda(\widehat{g}(\alpha)-1) \geq 0) .
$$

If $\widehat{g}\left(\alpha_{L}\right)<\infty$, then the Lundberg constant is a unique solution of the equation

$$
\alpha_{L} c-\lambda\left(\widehat{g}\left(\alpha_{L}\right)-1\right)=0 .
$$

Indeed, if $\widehat{g}\left(\alpha_{L}\right)<\infty$, then the supremum on the right hand side of (27)) is attained at the point $\alpha_{L}$, since $\widehat{g}(\alpha)$ is continuous for $\alpha \leq \alpha_{L}$. This implies (28). Further, the right hand side of (25) is a convex function and equals zero for $\alpha \in\left\{0, \alpha_{L}\right\}$. This proves the first statement of this remark.

Example 1. If the assumptions of Theorem 3 hold, then

$$
\sup _{0 \leq s \leq t \leq T} \sup _{x \geq 0, B \in \Xi}\left|P_{s x}\left(X_{t} \in B\right)-P_{s x}\left(\bar{X}_{t} \in B\right)\right| \leq \int_{0}^{T}\left|\lambda_{u}-\lambda\right| d u .
$$

It is necessary to set $\alpha=0$ in Theorem 3 to check (29). 
Theorem 4. Let $\left(X_{t}, t \geq 0\right)$ be a nonhomogeneous risk process whose infinitesimal operator is given by (21) and let $\left(\bar{X}_{t}\right)$ be the corresponding homogeneous process whose intensity $\lambda$ is such that the nondegeneracy condition (24) holds.

If inequality (25) holds for the constant $\alpha \geq 0$, then

$$
\begin{aligned}
e_{\alpha} & \equiv \sup _{x \geq 0} \exp (\alpha x)\left|P_{0 x}\left(\tau_{x}<\infty\right)-P_{0 x}\left(\bar{\tau}_{x}<\infty\right)\right| \\
& \leq \widehat{g}(\alpha) \int_{0}^{\infty}\left|\lambda_{u}-\lambda\right| \exp \left(-\beta u+(\alpha c-\beta) \int_{0}^{u}\left(\lambda_{v}-\lambda\right) / \lambda d v\right) d u .
\end{aligned}
$$

Example 2. If $\alpha=0$, then $\beta=0$ and inequality (30) implies

$$
\sup _{x \geq 0}\left|P_{0 x}\left(\tau_{x}<\infty\right)-P_{0 x}\left(\bar{\tau}_{x}<\infty\right)\right| \leq \int_{0}^{\infty}\left|\lambda_{u}-\lambda\right| d u .
$$

Example 3. Let $\lambda_{u}=\lambda+\varepsilon \sin u, 0 \leq \varepsilon<\lambda$. Then

$$
e_{\alpha} \leq \varepsilon \widehat{g}(\alpha) \exp (2 \varepsilon(\alpha c-\beta) / \lambda) / \beta
$$

for all $\alpha \in\left(0, \alpha_{L}\right)$, since

$$
\begin{aligned}
e_{\alpha} & \leq \widehat{g}(\alpha) \int_{0}^{\infty} \varepsilon|\sin u| \exp (-\beta u+(\alpha c-\beta)(1-\cos u) / \lambda) d u \\
& \leq \widehat{g}(\alpha) \int_{0}^{\infty} \varepsilon \exp (-\beta u+(\alpha c-\beta) 2 / \lambda) d u
\end{aligned}
$$

in view of inequality (30).

Example 4. Let $\lambda_{u}=\lambda-\varepsilon \exp (-u), 0 \leq \varepsilon<\lambda$. Then

$$
e_{\alpha} \leq \varepsilon \widehat{g}(\alpha) /(1+\beta)
$$

for all $\alpha \in\left[0, \alpha_{L}\right]$, since

$$
\begin{aligned}
e_{\alpha} & \leq \widehat{g}(\alpha) \int_{0}^{\infty} \varepsilon e^{-u} \exp \left(-\beta u-(\alpha c-\beta)\left(1-e^{-u}\right) / \lambda\right) d u \\
& \leq \widehat{g}(\alpha) \int_{0}^{\infty} \varepsilon \exp (-u-\beta u) d u=\varepsilon \widehat{g}(\alpha) /(1+\beta)
\end{aligned}
$$

according to inequality (30).

Example 5. Let $\alpha=\alpha_{L}>0, \widehat{g}(\alpha)<\infty$, and let the Lundberg constant for the homogeneous process be equal to

$$
K(\alpha) \equiv \lim _{x \rightarrow \infty} \exp (\alpha x) P_{0 x}\left(\bar{\tau}_{x}<\infty\right) .
$$

If

$$
\delta \equiv \int_{0}^{\infty}\left|\lambda_{u}-\lambda\right| d u<\infty
$$

then

$$
\left|\varlimsup_{x \rightarrow \infty}(\underline{\lim }) \exp (\alpha x) P_{0 x}\left(\tau_{x}<\infty\right)-K(\alpha)\right| \leq \widehat{g}(\alpha) \frac{\lambda}{\alpha c}\left[\exp \left(\delta \frac{\alpha c}{\lambda}\right)-1\right]
$$

and

$$
\text { the limit } \lim _{x \rightarrow \infty} x^{-1} \ln P_{0 x}\left(\tau_{x}=\infty\right)=\alpha_{L} \quad \text { exists }
$$

for all sufficiently small $\delta$. 
For the proof of (36) and (37), we use the remark to Theorem 3 implying for $\alpha=\alpha_{L}$ that inequality (25) becomes the equality (28) in this case, that is, $\beta=0$. Changing the function $\lambda_{v}-\lambda$ in (30) by its absolute value we get the inequality

$$
e_{\alpha} \leq \widehat{g}(\alpha) \int_{0}^{\infty}\left|\lambda_{u}-\lambda\right| \exp \left(\alpha c \int_{0}^{u}\left|\lambda_{v}-\lambda\right| / \lambda d v\right) d u .
$$

Then we change the variable $x=\int_{0}^{u}\left|\lambda_{v}-\lambda\right| d v$, so that

$$
e_{\alpha} \leq \widehat{g}(\alpha) \frac{\lambda}{\alpha c}\left[\exp \left(\frac{\alpha c}{\lambda} \int_{0}^{\infty}\left|\lambda_{u}-\lambda\right| d u\right)-1\right] .
$$

On the other hand, the definition of $e_{\alpha}$ in (30) yields for all $x \geq 0$ that

$$
-e_{\alpha} \leq e^{\alpha x} P_{0 x}\left(\tau_{x}<\infty\right)-e^{\alpha x} P_{0 x}\left(\bar{\tau}_{x}<\infty\right) \leq e_{\alpha} .
$$

Taking the supremum and infimum as $x \rightarrow \infty$ we derive inequality (36) from the latter results and (38).

Finally, inequality (36) implies that $\underline{\lim }_{x \rightarrow \infty} \exp (\alpha x) P_{0 x}\left(\tau_{x}<\infty\right)$ is positive and $\varlimsup_{x \rightarrow \infty} \exp (\alpha x) P_{0 x}\left(\tau_{x}<\infty\right)$ is finite for all sufficiently small $\int_{0}^{\infty}\left|\lambda_{u}-\lambda\right| d u$. Therefore equality (37) holds.

\section{Proofs}

Proof of Theorem 1. We use Theorem 1 of [5]. Note that assumptions (A.P), (D), and $(\mathrm{T})$ of [5] are identical to the corresponding assumptions of this paper, while assumption (Q) of [5] holds in view of (9). According to Theorem 1 of [5],

$$
\mu P_{s t} f=\mu Q_{t-s} f+\int_{s}^{t} \mu P_{s u} D_{u} Q_{t-u} f d u
$$

for all $\mu \in \aleph, f \in \Im$, and all $0 \leq s \leq t$.

Consider the operator

$$
\Delta_{s t}^{\beta} \equiv \exp (\beta(t-s))\left(P_{s t}-Q_{t-s}\right) .
$$

We deduce from (39) that

$$
\begin{aligned}
\mu \Delta_{s t}^{\beta} f & =\int_{s}^{t} e^{\beta(u-s)} \mu P_{s u} D_{u} Q_{t-u} f e^{\beta(t-u)} d u \\
& =\int_{s}^{t} e^{\beta(u-s)} \mu Q_{s u} D_{u} Q_{t-u} f e^{\beta(t-u)} d u+\int_{s}^{t} \mu \Delta_{s u}^{\beta} D_{u} Q_{t-u} f e^{\beta(t-u)} d u .
\end{aligned}
$$

This together with (13) implies that

$$
\left|\mu \Delta_{s t}^{\beta} f\right| \leq\|\mu\| q_{T}(\beta) \varepsilon_{T}(\beta)\|f\|+\sup _{s \leq u \leq T}\left\|\mu \Delta_{s u}^{\beta}\right\| \varepsilon_{T}(\beta)\|f\|
$$

for all $0 \leq s \leq t \leq T$.

Taking the supremum on both sides of the latter inequality over $f \in \Im_{0}$ such that $\|f\| \leq 1$ and then over $s \leq t \leq T$ we obtain

$$
\sup _{s \leq u \leq T}\left\|\mu \Delta_{s u}^{\beta}\right\| \leq\|\mu\| q_{T}(\beta) \varepsilon_{T}(\beta)+\sup _{s \leq u \leq T}\left\|\mu \Delta_{s u}^{\beta}\right\| \varepsilon_{T}(\beta),
$$

whence

$$
\sup _{s \leq u \leq T}\left\|\mu \Delta_{s u}^{\beta}\right\| \leq\|\mu\| q_{T}(\beta) \varepsilon_{T}(\beta) /\left(1-\varepsilon_{T}(\beta)\right) .
$$

Now we evaluate the supremum in the latter relation over all $\mu$ such that $\|\mu\|=1$ and prove inequality (15). 
M. V. KARTASHOV

Proof of Theorem 2. We substitute $f=1$ in (39) and approach the limit as $t \rightarrow \infty$. Then we obtain

$$
\mu p_{s}-\mu \bar{p}=\int_{s}^{\infty} \mu P_{s u} D_{u} \bar{p} d u
$$

by the monotone convergence theorem.

This proves (17).

Proof of Theorem 3. Consider the space $(E, \Xi)=\left(\mathbb{R}_{+}, \mathfrak{B}\left(\mathbb{R}_{+}\right)\right)$.

For all $\alpha \geq 0$, we define the Banach spaces $\aleph(\alpha)$ and $\Im(\alpha)$ of $\sigma$-finite charges

$$
\aleph(\alpha)=\left\{\mu:\|\mu\|_{\alpha} \equiv \int_{0}^{\infty} \exp (-\alpha x)|\mu|(d x)<\infty\right\}
$$

and bounded $\Xi$-measurable functions

$$
\Im(\alpha)=\left\{f:\|f\|_{\alpha} \equiv \sup _{x \in E} \exp (\alpha x)|f(x)|<\infty\right\},
$$

respectively.

The corresponding operator norm of the transition kernel $Q(x, B)$ equals

$$
\|Q\|_{\alpha}=\sup _{x \in E} \int_{0}^{\infty} \exp (\alpha(x-y))|Q|(x, d y)
$$

In particular, this implies that

$$
\begin{aligned}
\left\|P_{s t}\right\|_{\alpha} & =\sup _{x \in E} \int_{0}^{\infty} \exp (\alpha(x-y)) P_{s t}(x, d y)=\sup _{x \in E} \mathrm{E}_{s x} \exp \left(\alpha\left(x-X_{t}\right)\right) \\
& =\exp \left(-\alpha c(t-s)+\Lambda_{s}^{t}(\widehat{g}(\alpha)-1)\right),
\end{aligned}
$$

where we used the definition of the compound Poisson process and equalities (19).

The estimate for $\left\|Q_{t-s}\right\|_{\alpha}$ is similar, but $\lambda(t-s)$ is substituted for $\Lambda_{s}^{t}$ in this case:

$$
\left\|Q_{t-s}\right\|_{\alpha} \leq \exp (-\alpha c(t-s)+\lambda(t-s)(\widehat{g}(\alpha)-1)) .
$$

A simple estimate (45) for the norm of the perturbed operator is available in the case under consideration. We obtained equality (41) in the proof of Theorem 1. Now we use (41) instead of the general but less sharp inequality of Theorem 1 and obtain the bound

$$
\left\|\Delta_{s t}^{\beta}\right\|_{\alpha} \leq \int_{s}^{t} e^{\beta(u-s)}\left\|P_{s u}\right\|_{\alpha}\left\|D_{u} Q_{t-u}\right\|_{\alpha} e^{\beta(t-u)} d u .
$$

The left hand side of (26) does not exceed

$$
\sup _{x \in E} \int_{0}^{T} \exp (\alpha(x-y)+\beta(t-s))\left|\left(P_{s t}-Q_{t-s}\right)\right|(x, d y)=\left\|\Delta_{s t}^{\beta}\right\|_{\alpha}
$$

by definition (40) and in view of equality (44). Thus we need to estimate the right hand side of (47).

We derive from the definition of the infinitesimal operator (21) and the definition of the perturbation $(\mathbf{A D})$ that

$$
D_{u} f(x)=\left(\lambda_{u}-\lambda\right)\left(\int_{-\infty}^{x} f(x-y) d G(y)-f(x)\right)
$$

for $f \in \Im_{0}(\alpha) \equiv C_{1}\left(\mathbb{R}_{+}\right) \cap C_{b}\left(\mathbb{R}_{+}\right) \cap \Im(\alpha)$.

The expression in parentheses on the right hand side of the latter equality is a difference of two nonnegative operators. Thus (44) implies that

$$
\left\|D_{u}\right\|_{\alpha} \leq\left|\lambda_{u}-\lambda\right| \max (\widehat{g}(\alpha), 1)=\left|\lambda_{u}-\lambda\right| \widehat{g}(\alpha) .
$$


Therefore the integrand in the integral on the right hand side of (47) does not exceed

$$
\begin{aligned}
\left|\lambda_{u}-\lambda\right| \widehat{g}(\alpha) \exp \left[\beta(u-s)-\alpha c(u-s)+\Lambda_{s}^{u}(\widehat{g}(\alpha)-1)-\alpha c(t-u)\right. & +\lambda(u-s)(\widehat{g}(\alpha)-1)+\beta(t-u)] \\
=\left|\lambda_{u}-\lambda\right| \widehat{g}(\alpha) \exp \left[\beta(t-s)-\alpha c(t-s)+\left(\Lambda_{s}^{u}-\lambda(u-s)\right)(\widehat{g}(\alpha)-1)\right. & \quad+\lambda(t-s)(\widehat{g}(\alpha)-1)] \\
=\left|\lambda_{u}-\lambda\right| \widehat{g}(\alpha) \exp \left[\left(\Lambda_{s}^{u}-\lambda(u-s)\right)(\beta-\alpha c) / \lambda\right], &
\end{aligned}
$$

where we used the definition (25) of the constant $\beta$.

Substituting (45), (46), and (49) into (47) we obtain inequality (26) with the help of (50).

Proof of Theorem 4. According to definition (16) of the functions $p_{s}$ and $\bar{p}$,

$$
\begin{gathered}
p_{s}(x)=\lim _{t \rightarrow \infty} P_{s x}\left(\tau_{x}>t, X_{t} \in E\right)=P_{s x}\left(\tau_{x}=\infty\right), \\
\bar{p}(x)=P_{0 x}\left(\bar{\tau}_{x}=\infty\right) .
\end{gathered}
$$

It is obvious that $p_{0}-\bar{p}$ equals the difference of ruin probabilities in (30), while the left hand side of (30) coincides with the norm $\left\|p_{0}-\bar{p}\right\|_{a}$.

To estimate the latter norm we apply inequality (17):

$$
\left\|p_{0}-\bar{p}\right\|_{\alpha} \leq \int_{0}^{\infty}\left\|P_{0 u}\right\|_{\alpha}\left\|D_{u} \bar{p}\right\|_{\alpha} d u
$$

Lemma. The ruin function is such that

$$
\psi(x) \equiv 1-\bar{p}(x) \leq \exp (-\alpha x) \quad \text { for all } x \geq 0
$$

and for all $0 \leq \alpha \leq \alpha_{L}$.

Proof. We apply representation (1.9) of $\left[\underline{8}\right.$ with $R=\alpha_{L}, F=G$, and $\alpha=\lambda$. Then we get

$$
\begin{aligned}
\exp \left(\alpha_{L} x\right) \psi(x)= & \frac{\lambda}{c} \int_{x}^{\infty} \exp \left(\alpha_{L} x\right)(1-G(y)) d y \\
& +\frac{\lambda}{c} \int_{0}^{x} \exp \left(\alpha_{L} y\right) \psi(x-y)(1-G(y)) d y .
\end{aligned}
$$

Since $\exp \left(\alpha_{L} x\right) \leq \exp \left(\alpha_{L} y\right)$ and $\psi(x-y) \leq 1$, we obtain

$$
\exp \left(\alpha_{L} x\right) \psi(x) \leq \frac{\lambda}{c} \int_{x}^{\infty} \exp \left(\alpha_{L} y\right)(1-G(y)) d y=1,
$$

where the latter inequality follows from (28).

We continue the proof of Theorem 4. Using equality (48) we get

$$
D_{u} \bar{p}(x)=\left(\lambda_{u}-\lambda\right)\left(G(x)-1-\int_{-\infty}^{x} \psi(x-y) d G(y)+\psi(x)\right) .
$$

Now we apply (52) and get

$$
\left|D_{u} \bar{p}(x)\right| \leq \exp (-\alpha x)\left|\lambda_{u}-\lambda\right| \widehat{g}(\alpha)
$$

for all $\alpha \in\left[0, \alpha_{L}\right]$. 
The definition of the norm in the space $\Im(\alpha)$ and relations (51), (45), and (55) yield the bound

$$
\begin{aligned}
\left\|p_{0}-\bar{p}\right\|_{\alpha} & \leq \int_{0}^{\infty} \exp \left(-\alpha c u+\Lambda_{0}^{u}(\widehat{g}(\alpha)-1)\right)\left|\lambda_{u}-\lambda\right| \widehat{g}(\alpha) d u \\
& =\widehat{g}(\alpha) \int_{0}^{\infty}\left|\lambda_{u}-\lambda\right| \exp \left(-\alpha c u+\Lambda_{0}^{u}(\alpha c-\beta) / \lambda\right) d u \\
& =\widehat{g}(\alpha) \int_{0}^{\infty}\left|\lambda_{u}-\lambda\right| \exp \left(-\beta u+\left(\Lambda_{0}^{u}-\lambda u\right)(\alpha c-\beta) / \lambda\right) d u
\end{aligned}
$$

where $\beta$ is defined by (25). This proves inequality (30).

\section{ACKNOWLedgement}

The author is indebted to the referee for valuable comments.

\section{BIBLIOGRAPHY}

1. N. V. Kartashov, Strong Stable Markov Chains, VSP/TViMS, Utrecht, The Netherlands/Kiev, Ukraine, 1996. MR1451375 (99e:60150)

2. E. B. Dynkin, Theory of Markov Processes, Fizmatlit, Moscow, 1962; English transl., PrenticeHall, Inc., Englewood Cliffs, N.J., 1961. MR0131900 (24:A1747)

3. I. I. Gikhman and A. V. Skorokhod, The Theory of Stochastic Processes, vol. 2, "Nauka", Moscow, 1973; English transl., Springer-Verlag, New York-Heidelberg, 1975. MR0341540 (49:6288) MR0375463(51:11656)

4. T. Kato, Perturbation Theory for Linear Operators, Springer-Verlag, Berlin-New York, 1966. MR0203473(34:3324)

5. M. V. Kartashov, On the stability of almost time-homogeneous Markov semigroups of operators, Teor. Imovir. Mat. Stat. 71 (2004), 105-113; English transl. in Theory Probab. Math. Statist. 72 (2005), 119-128. MR2144325 (2006b:60172)

6. M. V. Kartashov, Ergodicity and stability of quasihomogeneous Markov semigroups of operators, Teor. Imovir. Mat. Stat. 72 (2005), 54-62; English transl. in Theory Probab. Math. Statist. 72 (2006), 59-68. MR2168136 (2006g:47065)

7. M. V. Kartashov and O. M. Stroev, The Lundberg approximation for the risk function in an almost homogeneous environment, Teor. Imovir. Mat. Stat. 73 (2005), 63-71; English transl. in Theory Probab. Math. Statist. 73 (2006), 71-79. MR2213842(2007b:62121)

8. J. Grandell, Aspects of Risk Theory, Springer Series in Statistics, Springer, 1991. MR.1084370 (92a:62151)

Department of Probability Theory and Mathematical Statistics, Faculty for Mechanics and Mathematics, National Taras Shevchenko University, Academician Glushkov Avenue, 6, KYIV 03127, UKRAINE

E-mail address: winf@ln.ua

Received 19/DEC/2005

Translated by S. KVASKO 